\title{
Optical-Resolution Photoacoustic Microscopy for Volumetric and Spectral Analysis of Histological and Immunochemical Samples**
}

\section{Yu Shrike Zhang ${ }^{\dagger}$,}

The Wallace H. Coulter Department of Biomedical Engineering, Georgia Institute of Technology and Emory University, Atlanta, GA 30332 (USA)

Junjie Yao ${ }^{\dagger}$,

Department of Biomedical Engineering, Washington University in St. Louis, St. Louis, MO 63130 (USA)

\section{Chi Zhang,}

Department of Biomedical Engineering, Washington University in St. Louis, St. Louis, MO 63130 (USA)

\section{Lei Li,}

Department of Biomedical Engineering, Washington University in St. Louis, St. Louis, MO 63130 (USA)

\section{Lihong V. Wang*, and}

Department of Biomedical Engineering, Washington University in St. Louis, St. Louis, MO 63130 (USA)

\section{Younan Xia*}

The Wallace H. Coulter Department of Biomedical Engineering Georgia Institute of Technology and Emory University, Atlanta, GA 30332 (USA)

School of Chemistry and Biochemistry, Georgia Institute of Technology, Atlanta, GA 30332 (USA)

\section{Abstract}

Optical-resolution photoacoustic microscopy (OR-PAM) is an imaging modality with superb penetration depth and excellent absorption contrast. Here we demonstrate, for the first time, that this technique can advance quantitative analysis of conventional chromogenic histochemistry. Because OR-PAM can quantify the absorption contrast at different wavelengths, it is feasible to spectrally resolve the specific biomolecules involved in a staining color. Furthermore, the

\footnotetext{
** This work was supported in part by NIH grants DP1 OD000798 (NIH Director's Pioneer Award) to Y. Xia, and DP1 EB016986 (NIH Director's Pioneer Award), R01 CA186567 (NIH Director's Transformative Research Award), and R01 CA159959 to L. V. Wang. L. V. Wang has a financial interest in Endra, Inc., and Microphotoacoustics, Inc., which, however, did not support this work. Part of the research was performed at the Alafi Neuroimaging Laboratory, the Hope Center for Neurological Disorders in Washington University School of Medicine, which is supported by the NIH Neuroscience Blueprint Center Core Grant P30 NS057105.

*younan.xia@bme.gatech.edu (for scaffold fabrication and histochemistry); and lhwang@ biomed.wustl.edu (for photoacoustic imaging and data analysis).

${ }^{\dagger}$ Y. S. Zhang and J. Yao contributed equally to this work.

Supporting information for this article is available on the WWW under http://dx.doi.org/.
} 
tomographic capability of OR-PAM allows for non-invasive volumetric imaging of a thick sample without microtoming it. By immunostaining the sample with different chromogenic agents, we further demonstrated the ability of OP-PAM to resolve different types of cells in a co-culture sample with imaging depths up to $1 \mathrm{~mm}$. Taken together, the integration of OR-PAM with (immuno)histochemistry offers a simple and versatile technique with broad applications in cell biology, pathology, tissue engineering, and related biomedical studies.

\section{Keywords}

photoacoustic microscopy; biomedical imaging; histochemistry; cell biology; tissue engineering

As a technique for analyzing the microscopic anatomy of biological tissues, histology is typically performed by sectioning fixed tissues into thin slices with a microtome, followed by staining with chromogenic dyes and then examination under an optical microscope. Although this technique has been serving biology, medicine, and bioengineering as an invaluable tool for more than one century, there is still plenty of room for improvement. For example, the sample has to be fixed and microtomed into thin slices of only a few micrometers thick before it can be stained and examined by conventional optical microscopy. It is difficult to identify and quantify a specific component of interest in a histology image unless this component can be somehow singled out manually using specialized software. As a result, histology analysis is always labor-intensive and timeconsuming. In addition, it is very challenging to generate three-dimensional (3D) reconstruction of a sample during histology analysis. ${ }^{[1]}$ With the help of confocal and multiphoton microscopy, it is now possible to perform 3D imaging and analysis but the samples have to be stained with special fluorescent dyes that tend to be more susceptible to photobleaching than chromogenic dyes involved in histochemical staining. Because of the strong light scattering from biological tissues, the penetration depths of these imaging modalities are often limited to a few hundred micrometers. ${ }^{[2]}$ The imaging depth further drops below $200 \mu \mathrm{m}$ when a highly scattering structure (e.g., a polymer scaffold) is involved. Here we demonstrate that all these issues could be addressed by using opticalresolution photoacoustic microscopy (OR-PAM) to analyze the stained samples directly.

As a newly developed technique, OR-PAM allows for non-invasive, three-dimensional (3D) imaging with relatively high spatial resolution and deep penetration. ${ }^{[3]}$ In OR-PAM, a nanosecond pulsed laser beam is focused and delivered into a biological sample to generate wide-band photoacoustic waves via the photoacoustic (PA) effect. The PA waves are then collected by an ultrasonic transducer moving around the sample (Fig. S1 in the Supporting Information). A 3D tomographic image can be readily constructed by raster scanning the sample and piecing together the depth-resolved one-dimensional (1D) images (A-lines). Unlike confocal and multi-photon microscopy, OR-PAM can be used for volumetric imaging without depth scanning, greatly shortening the image acquisition time. OR-PAM can reach an imaging depth of $c a .1 \mathrm{~mm}$ while maintaining a lateral resolution of $c a .5 \mu \mathrm{m}$, which is on the same scale as cells. OR-PAM has found increasing applications in biomedical research by working with intrinsic optical absorption contrast agents including hemoglobin and melanin or external contrast agents such as indocyanine green (ICG) and 
metal nanoparticles. ${ }^{[3,4]}$ As recently demonstrated by our groups, the capability of OR-PAM can be extended to image essentially all types of living cells by staining the cells with a metabolic dye. ${ }^{[5]}$ Here we further establish that OR-PAM can also be used for volumetric and spectral examination of histological samples with a minimum effort for either sample preparation or image analysis.

We first demonstrated the feasibility of using OR-PAM to directly analyze the samples stained by the traditional histological dyes. Figure 1a shows a representative transmission optical micrograph of a mouse connective tissue slice ( $c a .5 \mu \mathrm{m}$ thick) stained with Masson's trichrome, where the erythrocytes (red blood cells), cytoplasm, and collagen fibrils were stained bright red, pinkish purple, and blue, respectively. From this optical micrograph, however, it was very difficult to single out a specific component such as collagen for further analysis. In contrast, by using OR-PAM and taking advantage of the unique absorption spectrum of each dye, the three different components could be readily separated from each other. As shown in Figure 1b, the PA signals from the stained erythrocytes decreased remarkably when the laser wavelength was tuned from 500 to 550 and then $590 \mathrm{~nm}$. In comparison, the stained collagen showed an opposite trend, with weak PA signals at $500 \mathrm{~nm}$ but much stronger PA signals at $550 \mathrm{~nm}$ and $590 \mathrm{~nm}$. The stained cytoplasm showed relatively uniform PA signals at all three wavelengths. Figure 1, c-e, shows OR-PAM maximum amplitude projection (MAP) images of the same sample acquired at 500, 550, and $590 \mathrm{~nm}$, respectively. Three sets of arrowheads with different colors were used to indicate the erythrocytes, cytoplasm, or collagen at the same locations in the images. At $500 \mathrm{~nm}$, erythrocytes gave the strongest PA signals, followed by cytoplasm, while collagen was barely detectable (Fig. 1c). At $550 \mathrm{~nm}$, all the three components showed similar PA amplitudes (Fig. 1d). The erythrocytes were not detectable at $590 \mathrm{~nm}$ (Fig. 1e). As shown in Figure 1f, all these three components could be easily differentiated in a combined PAM MAP image after spectral analysis (see Experimental section in the Supporting Information for details). It should be pointed out that the superimposed PAM MAP image in Figure 1f might suffer certain degree of loss in detail as compared to Figure 1a. This issue can be addressed by taking PAM images with a greater signal-to-noise ratio to obtain more accurate spectral analysis, as well as using a system with a higher lateral resolution. Based on the PAM data, we could further quantify the area occupied by each component. In Figure 1f, the areas occupied by erythrocytes, cytoplasm, and collagen were 5717,90097 , and $134190 \mu \mathrm{m}^{2}$, respectively, corresponding to a ratio of 1:15.8:23.5. These results demonstrate that OR-PAM can significantly reduce the amount of time required for image analysis while ensuring a desired quality for the image. The same strategy is also applicable to other histological staining with multiple colors, including the hematoxylin and eosin staining that gives cytoplasm and nuclei pinkish red and blue colors, respectively. As shown in Figure S1a, eosin has a sharp absorption peak at $c a .520 \mathrm{~nm}$ whereas hematoxylin exhibits a broad peak extending from $<400 \mathrm{~nm}$ all the way to $650 \mathrm{~nm}$. Accordingly, using a dual-wavelength PAM scanning at $523 \mathrm{~nm}$ and $587 \mathrm{~nm}$, we could easily and effectively differentiate the hematoxylin-stained nuclei from the eosin-stained cytoplasm in the same image (Fig. S1, c-e). 
In addition to traditional histology, we further extended OR-PAM to immunostaining in an effort to achieve better selectivity. For immunofluorescence-based histology, our own experience suggests that a laser scanning confocal microscope can only reach a maximum imaging depth of $c a .100 \mu \mathrm{m}$ if the cells are embedded in a highly scattering scaffold made of a polymer such as poly(D,L-lactide-co-glycolide) (PLGA). In comparison, by switching to a combination of OR-PAM and chromogenic immunochemistry, we can at least double this penetration depth to 200-300 $\mu \mathrm{m}$, together with a lateral resolution of $c a$. $5 \mu \mathrm{m}$, thanks to the one-way optical scattering (and the much weaker return acoustic scattering) by the sample during imaging. Figure 2a shows a UV-vis spectrum of the reaction product of horseradish peroxidase (HRP) and ImmPACT diaminobenzidine (DAB) peroxidase substrate, which has a strong absorption peak at $320 \mathrm{~nm}$, in addition to a broad peak extending from $\mathrm{ca}$. $400 \mathrm{~nm}$ to $900 \mathrm{~nm}$. Figure $2 \mathrm{~b}$ shows a transmission optical micrograph of MC3T3 preosteoblasts whose f-actin was stained in sequence with biotin-phalloidin and streptavidin-HRP, followed by color development with DAB. Figure 2c shows an OR-PAM MAP image captured at $570 \mathrm{~nm}$ from a different region of the same sample, clearly revealing both the stained cytoskeleton and the unstained nuclei. This result demonstrates the ability of OR-PAM to resolve the specific components of a cell after immunostaining.

We then seeded MC3T3 pre-osteoblasts in a PLGA inverse opal scaffold with uniform pores. ${ }^{[4 \mathrm{e}, 4 \mathrm{~h}, 4 \mathrm{k}, 5,6]}$ Figure $2 \mathrm{~d}$ shows a transmission optical micrograph of cells that were cultured in the scaffold for 3 days and then stained for $\mathrm{f}$-actin in sequence with biotinphalloidin, streptavidin-HRP, and DAB. The cells typically stretched along the walls of the pores. As shown in the OR-PAM MAP image (Fig. 2e), the same pattern of cell attachment was also observed. In addition, we could reconstruct the 3D distribution of the stained cells in the scaffold (Movie S1). We noticed that the cells at 100-200 $\mu \mathrm{m}$ below the top surface of the scaffold also showed up in the MAP image (Fig. 2e) and the PA signals from these cells could be easily separated from the rest of the sample (Fig. 2f) based on the volumetric information provided by PAM. In comparison, it was very difficult to resolve these cells under a transmission optical microscope (Fig. 2d) unless the scaffold was physically sectioned into thin slices prior to imaging. For such scaffold-based samples, OR-PAM can easily reach a penetration depth of 200-300 $\mu \mathrm{m}$, which is more than twice that of confocal microscopy. The penetration depth can be further doubled by imaging the sample from both the top and bottom sides. ${ }^{[7]}$ At the expense of spatial resolution, PA imaging can even achieve a penetration depth of a few centimeters. ${ }^{[8]}$ Furthermore, PA imaging is essentially background-free because OR-PAM relies on optical absorption and cells, cellular components, extracellular matrix (ECM) molecules, and biomaterials with non-absorption at the laser wavelength should not contribute to the PA signals. This important feature of PAM holds its superiority to fluorescence-based imaging modalities (e.g., confocal and multiphoton microscopy) especially when observing tissue-level samples because certain ECM molecules (or scaffolds) such as collagen can give strong autofluorescence signals in a broad range of wavelengths, greatly limiting the selection of fluorescent dyes. ${ }^{[9]}$

OR-PAM can also be used to differentiate cells enhanced by chromogenic immunostaining. This capability is made possible by the recent development of various chromogenic substrates that present distinctive absorption spectra after reacting with HRP, including 
ImmPACT DAB (brown), NovaRed (red), and Vector SG (blue/gray). ${ }^{[10]}$ In our study, we chose ImmPACT DAB and NovaRed because the absorption spectra of their reaction products with HRP fit the wavelengths currently used in our PAM. Figure 3a shows the absorption spectra of reaction products of HRP with ImmPACT DAB and NovaRed, respectively. The product of $\mathrm{DAB}$ chromogen had a distinct absorption peak centered at $c a$. $500 \mathrm{~nm}$, whereas both products showed similar absorptions at $>570 \mathrm{~nm}$. The photographs in the inset of Figure 3a clearly show the different colors of the two chromogenic products. We attempted to distinguish cell populations in a sample co-cultured with MC3T3 preosteoblasts and SK-BR-3 human breast tumor cells. Figure 3b shows a typical reflection optical micrograph of the sample hosted on a glass slide. The f-actins of both types of cells were stained consecutively with biotin-phalloidin, streptavidin-HRP, and DAB (brown), whereas the SK-BR-3 cells were further stained in sequence with anti-HER2 primary antibody (specific to human), HRP-secondary antibody, and NovaRed (dark red) for HER2 antigens on their surfaces. Unlike MC3T3 cells that assumed highly stretched shapes with large areas, SK-BR-3 cells were characterized by their relatively small sizes and less stretched shapes. Figure $3 \mathrm{c}$ shows another transmission optical micrograph taken from a sample co-cultured in a PLGA inverse opal scaffold with a uniform pore size of $200 \mu \mathrm{m}$. The sample was then imaged by OR-PAM at dual wavelengths of $523 \mathrm{~nm}$ and $570 \mathrm{~nm}$. Based on the absorption spectra of the two staining dyes, SK-BR-3 cells could be spectrally unmixed from the background MC3T3 cells using custom-coded MATLAB programs (Fig. 3d, where SK-BR-3 cells are shown in yellow/green and MC3T3 cells in reddish brown). Volumetric rendering showing the two cell populations in the scaffold is shown in Movie S2. The exact positions of the SK-BR-3 cells could be determined in magnified PAM MAP images from topical and sagittal views (Fig. 3e). Volumetric distributions of individual SKBR-3 cells or their clusters could be clearly resolved across the entire thickness of the scaffold filled with MC3T3 cells, which is otherwise impossible with conventional optical microscopy without physically sectioning the sample (Fig. 3c). Details with regard to image processing can be found in the Experimental section in the Supporting Information.

In summary, we have successfully demonstrated that OR-PAM can be used for histology analysis in both two and three dimensions. Biological components stained with traditional chromogenic histological dyes can be spectrally separated in PAM imaging. In addition, we have shown that OR-PAM can image specific cell markers stained by antibody-based chromogenic dyes (i.e., immunohistochemistry). Taking together, we expect that OR-PAM can potentially become a potent alternative imaging technique for histology analysis in biomedical applications, including but not limited to, cell biology, pathology, tissue engineering, and regenerative medicine.

\section{Supplementary Material}

Refer to Web version on PubMed Central for supplementary material.

\section{References}

1. Song Y, Treanor D, Bulpitt AJ, Magee DR. J. Pathol. Inform. 2013; 4:7. [PubMed: 23869286]

2. Dumas, D.; Riquelme, B.; Werkmeister, E.; Isla, ND.; Stoltz, JF. Multimodality of microscopy imaging applied to cartilage tissue engineering. Amsterdam: IOS Press; 2007. 
3. a) Maslov K, Zhang HF, Hu S, Wang LV. Opt. Lett. 2008; 33:929-931. [PubMed: 18451942] b) Hu S, Maslov K, Wang LV. Opt. Lett. 2011; 36:1134-1136. [PubMed: 21479007]

4. a) Razansky D, Distel M, Vinegoni C, Ma R, Perrimon N, Koster RW, Ntziachristos V. Nat. Photonics. 2009; 3:412-417.b) Wang LV. Nat. Photonics. 2009; 3:503-509. [PubMed: 20161535] c) Jin Y, Jia C, Huang SW, O’Donnell M, Gao X. Nat. Commun. 2010; 1:41. [PubMed: 20975706] d) Kim C, Favazza C, Wang LV. Chem. Rev. 2010; 110:2756-2782. [PubMed: 20210338] e) Zhang Y, Cai X, Choi SW, Kim C, Wang LV, Xia Y. Biomaterials. 2010; 31:8651-8658. [PubMed: 20727581] f) Yao J, Maslov KI, Zhang Y, Xia Y, Wang LV. J. Biomed. Opt. 2011; 16:076003076011. [PubMed: 21806264] g) Wang LV, Hu S. Science. 2012; 335:1458-1462. [PubMed: 22442475] h) Cai X, Zhang Y, Li L, Choi SW, MacEwan MR, Yao J, Kim C, Xia Y, Wang LV. Tissue Eng. 2012; 19:196-204.i) Cai X, Zhang YS, Xia Y, Wang LV. Mater. Today. 2013; 16:6777.j) Zhang YS, Wang Y, Wang L, Wang Y, Cai X, Zhang C, Wang LV, Xia Y. Theranostics. 2013; 3:532-543. [PubMed: 23946820] k) Zhang YS, Cai X, Yao J, Xing W, Wang LV, Xia Y. Angew. Chemie. Int. Ed. 2014; 53:184-188.

5. Zhang Y, Cai X, Wang Y, Zhang C, Li S-W, Choi L, Wang LV, Xia Y. Angew. Chem. Int. Ed. 2011; 50:7359-7363.

6. a) Choi S-W, Zhang Y, Thomopoulos S, Xia Y. Langmuir. 2010; 26:12126-12131. [PubMed: 20450216] b) Choi S-W, Zhang Y, Xia Y. Langmuir. 2010; 26:19001-19006. [PubMed: 21090781] c) Zhang Y, Choi S-W, Xia Y. Macromol. Rapid Commun. 2012; 33:296-301. [PubMed: 22231861] d) Choi S-W, Zhang Y, MacEwan MR, Xia Y. Adv. Healthcare Mater. 2013; 2:145154.e) Zhang YS, Regan KP, Xia Y. Macromol. Rapid Commun. 2013; 34:485-491. [PubMed: 23365045] f) Zhang YS, Choi SW, Xia Y. Soft Matter. 2013; 9:9747-9754.

7. Yao J, Maslov KI, Puckett ER, Rowland KJ, Warner BW, Wang LV. Opt. Lett. 2012; 37:659-661. [PubMed: 22344139]

8. a) Ku G, Wang LV. Opt. Lett. 2005; 30:507-509. [PubMed: 15789718] b) Kim C, Erpelding TN, Jankovic L, Pashley MD, Wang LV. Biomed. Opt. Express. 2010; 1:278-284. [PubMed: 21258465]

9. a) Gareau DS, Bargo PR, Horton WA, Jacques SL. J. Biomed. Opt. 2004; 9:254-258. [PubMed: 15065888] b) Mansfield JR, Gossage KW, Hoyt CC, Levenson RM. J. Biomed. Opt. 2005; 10:41207. [PubMed: 16178631]

10. Discovery through color - a guide to multiple antigen labeling. Burlingame: Vector Laboratories; 2005.

11. a) Zhang C, Maslov KI, Wang LV. Opt. Lett. 2010; 35:3195-3197. [PubMed: 20890331] b) Zhang C, Zhang YS, Yao DK, Xia Y, Wang LV. J. Biomed. Opt. 2013; 18:020504.

12. a) Zhang HF, Maslov K, Sivaramakrishnan M, Stoica G, Wang LV. Appl. Phys. Lett. 2007; 90:053901.b) Wang Y, Hu S, Maslov K, Zhang Y, Xia Y, Wang LV. Opt. Lett. 2011; 36:1029. [PubMed: 21478972] 

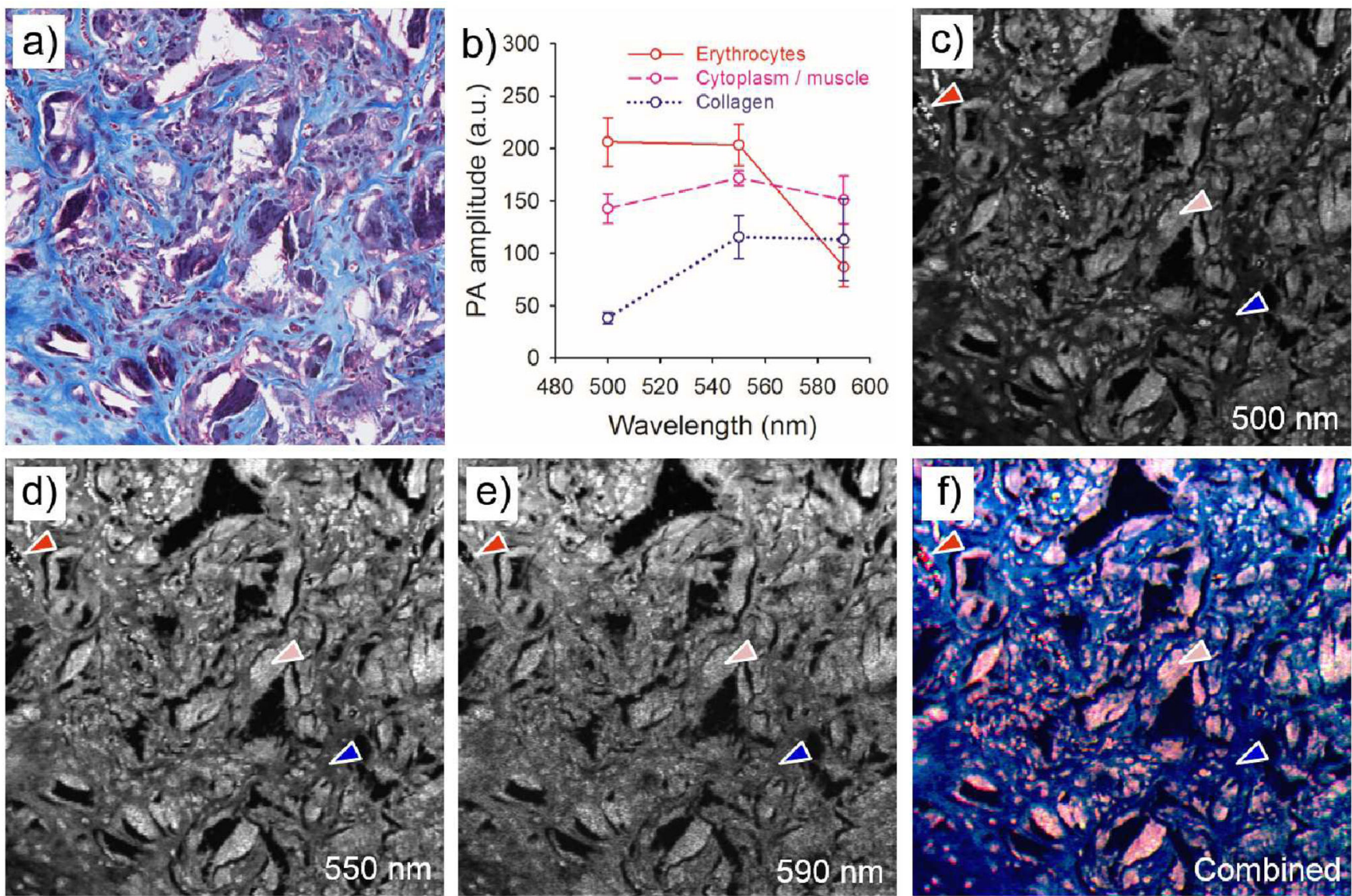

Min

Max PA amplitude (a.u.)

$100 \mu \mathrm{m}$

Figure 1.

a) A transmission optical micrograph of a histological slice of $5 \mu \mathrm{m}$ thick that was sectioned from mouse connective tissue and stained with Masson's trichrome. The erythrocytes, cytoplasm, and collagen were stained bright red, pinkish purple and blue, respectively. b) PA signals collected from the aforementioned three components at wavelengths of $500 \mathrm{~nm}$, $550 \mathrm{~nm}$ and $590 \mathrm{~nm}$. c-e) OR-PAM MAP images of the same sample acquired at wavelengths of $500 \mathrm{~nm}, 550 \mathrm{~nm}$ and $590 \mathrm{~nm}$, respectively. The arrowheads with three different colors indicate representative components of erythrocytes, cytoplasm, and collagen. f) Combined OR-PAM MAP image of the three components that were spectral decomposed. The same features could be observed as in (a). MAP, maximum amplitude projection. 

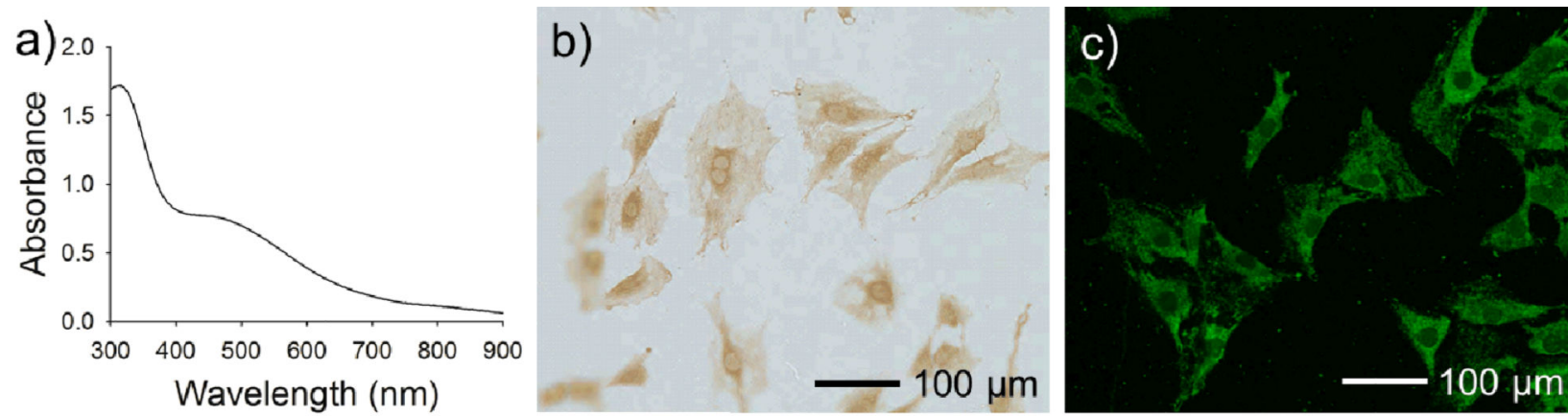

Max
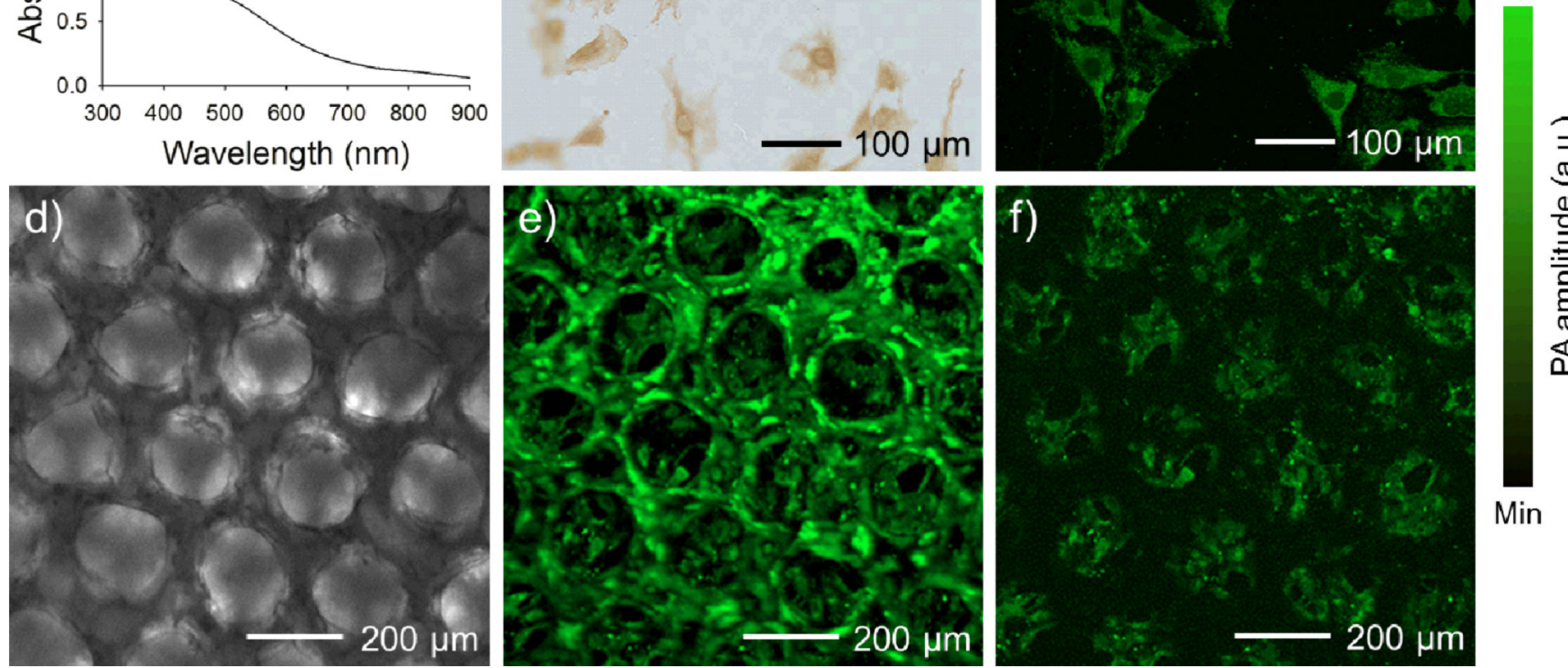

Figure 2.

a) UV-vis absorption spectrum of the reaction product of ImmPACT DAB and horseradish peroxidase (HRP). b) A transmission optical micrograph of MC3T3 cells stained for f-actin in sequence with biotin-phalloidin, streptavidin-HRP, and DAB. c) Submicron highresolution OR-PAM MAP image showing MC3T3 cells stained for f-actin with the same procedure as in (b). d) A transmission optical micrograph showing the cells cultured in an inverse opal scaffold for three days and stained with biotin-phalloidin/HRP-streptavidin/ DAB. e) OR-PAM MAP image of the same cell/scaffold construct in (d). f) OR-PAM image showing the bottom section (100-200 $\mu \mathrm{m}$ beneath the top surface) of the surface pores in the scaffold. Note that cell attachment and the structure of the pores can be clearly observed. MAP, maximum amplitude projection. 

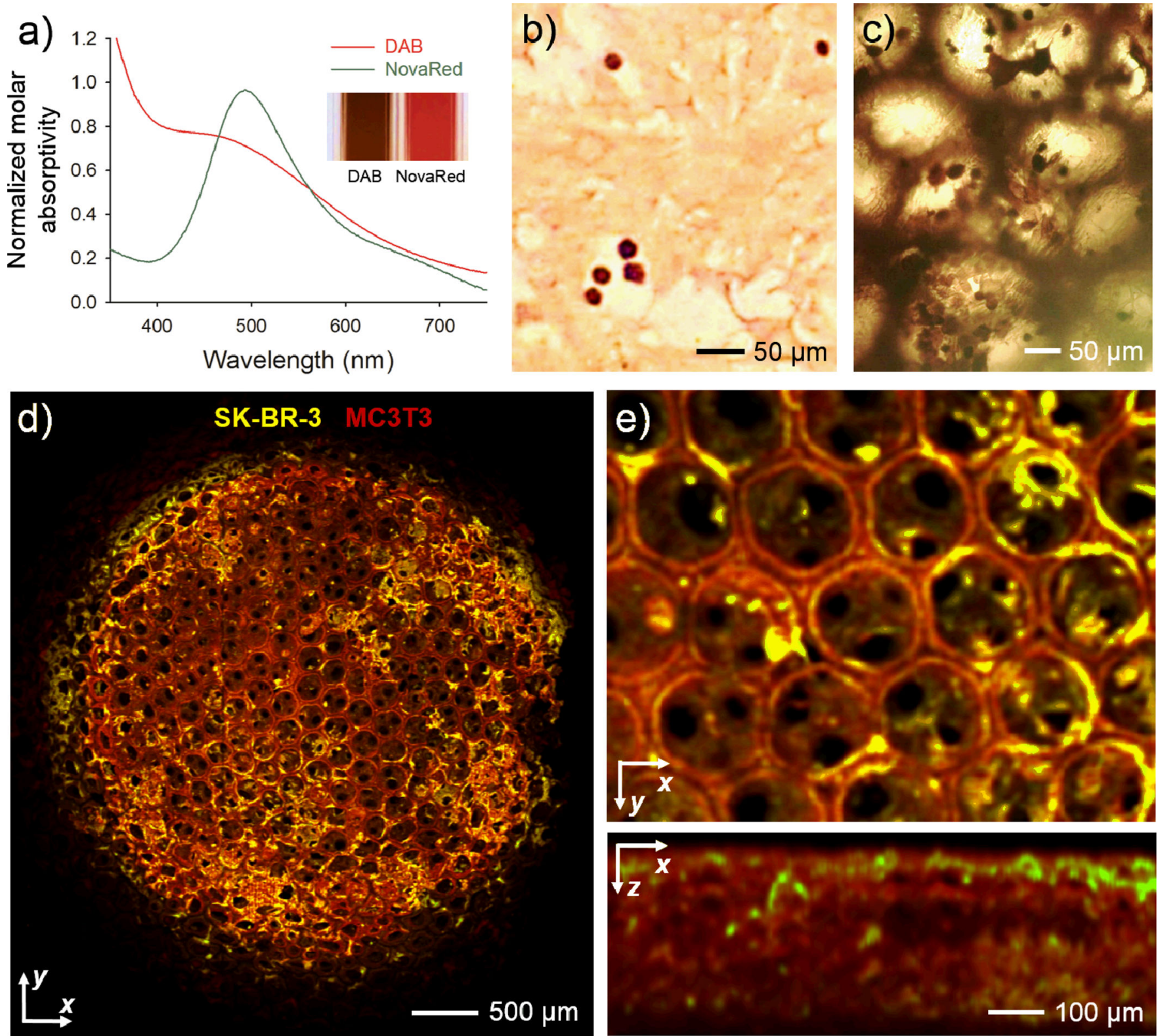

Min

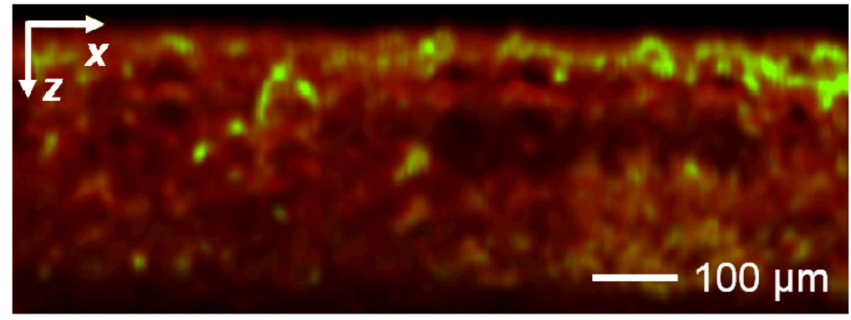

Max PA amplitude (a.u.)

Figure 3.

a) Normalized molar absorptivity of the reaction products of HRP with DAB and NovaRed, respectively. The inset displays optical micrographs of these products, clearly showing their different colors. b) An optical micrograph of a co-culture sample of SK-BR-3 and MC3T3 cells on a glass slide after staining with biotin-phalloidin/streptavidin-HRP/DAB for f-actin, and anti-HER2/HRP-secondary antibody/NovaRed for HER2 antigens on the surfaces of SK-BR-3 cells. The SK-BR-3 cells were shown in red while the MC3T3 cells in the background were shown in brown. c) An optical micrograph showing a co-culture sample in a PLGA inverse opal scaffold obtained by following the same staining procedure as in (b). d) OR-PAM MAP image of the same scaffold at a low magnification, where SK-BR-3 and 
MC3T3 cells could be digitally separated in the image using a custom-written spectral unmixing algorithm (see Experimental section in the Supporting Information for details). e) Magnified views of the same sample showing volumetric distribution of the two cell types from the top and side, respectively. MAP, maximum amplitude projection. 